\section{POS1191 COVID-19 STRESS: DOES IT AUGMENT PAIN IN PEOPLE WITH FIBROMYALGIA AND MIGHT PSYCHOLOGICAL FLEXIBILITY PROTECT AGAINST THIS INFLUENCE?}

T. Koppert ${ }^{1}$, J. W. G. Jacobs ${ }^{2}$, M. Lumley ${ }^{3}$, R. Geenen ${ }^{4,5} .{ }^{1}$ Leiden University Faculty of Social Sciences, Developmental \& Educational Psychology, Leiden, Netherlands; ${ }^{2}$ University Medical Center Utrecht, Rheumatology \& Clinical Immunology, Utrecht, Netherlands; ${ }^{3}$ Wayne State University, Psychology, Detroit, United States of America; ${ }^{4}$ Utrecht University, Psychology, Utrecht, Netherlands; ${ }^{5}$ Altrecht, Psychosomatic Medicine, Zeist, Netherlands

Background: Stress might augment pain in people with fibromyalgia, possibly through sensitization of the central nervous system. The COVID-19 pandemic offers a unique opportunity to examine this mechanism. If it holds, the link between COVID-19 stress and pain would be stronger in people with fibromyalgia than in people without it. Additionally, psychological flexibility might act as a resilience factor, reducing pain severity. If psychological flexibility buffers the impact of stress on pain in people with fibromyalgia, then enhancing psychological flexibility may be of value.

Objectives: To determine the association between COVID-19 stress and pain severity in people with fibromyalgia, as compared to people without fibromyalgia, and whether psychological flexibility buffers the impact of stress on pain severity. Methods: In a repeated cross-sectional design, we analysed questionnaire data from two independent surveys. The data collection has been described in a previous study of mental well-being in people with inflammatory rheumatic diseases. [1] The current study analysed data from people with and without fibromyalgia Data were collected before the COVID-19 pandemic (2018; fibromyalgia: $n=145$, no fibromyalgia: $n=386$ ) and at the first peak of the pandemic in the Netherlands (2020; fibromyalgia: $n=270$, no fibromyalgia: $n=1259$ ). Stress due to the pandemic, psychological flexibility, and pain were subjected to regression analyses. Two operationalisations of stress were analysed: self-reported stress levels during the peak of the pandemic in 2020, and a comparison of assessments in 2018 and 2020 (assuming higher stress levels during the pandemic peak in 2020). Results: In regression analyses, stress during the pandemic $(p<.001)$, having fibromyalgia $(p<.001)$, and lower psychological flexibility $(p<.001)$ were all associated with more severe pain, but the interactions showed that the strength of the association of stress with pain was not different in people with fibromyalgia compared to people without fibromyalgia ( $p=.76$ and $p=.28$ for the two operationalization of stress, respectively). Another interaction indicated that psychological flexibility was a potential buffer against the association between self-reported stress and pain in the first operationalisation of stress $(p=.04)$, but not in the second $(p=.44)$. The significant but small interaction is shown in the Figure 1. It suggests that pain is higher in people with low psychological flexibility and higher stress levels during the pandemic. This effect was not specific to fibromyalgia. Conclusion: Overall, the significant main effects show that negative states, such as stress related to the pandemic and low psychological flexibility, are associated with another negative state: pain. A small interaction effect suggests that psychological flexibility may protect against the impact of COVID-19 stress on pain, both in people with and without fibromyalgia. However, the analyses reject our hypothesis that COVID-19 stress would augment pain especially in people with fibromyalgia. REFERENCES:

[1] Koppert TY, Jacobs JWG, Geenen R. The psychological impact of the COVID-19 pandemic on Dutch people with and without an inflammatory rheumatic disease. Rheumatology (Oxford) 2020; keaa842. doi: 10.1093/ rheumatology/keaa842.

Pain (standard deviation from the norm) as a function of low (-1 SD) and high (+1 SD) psychological flexibility and low (-1 SD) and high (-1 SD) self-reported stress levels during the first peak of the pandemic in 2020, while controlling for gender, age, education level, and number of diseases.

Disclosure of Interests: Tim Koppert: None declared, Johannes W.G. Jacobs: None declared, Mark Lumley Consultant of: Paid research consultant to Cognifisense, LLP, which studies virtual reality treatments for chronic pain., Rinie Geenen Speakers bureau: Sanofi Genzyme paid for a lecture and worshop on depression and fatigue in RA.

DOI: 10.1136/annrheumdis-2021-eular.1529

\section{POS1192 \\ BEHAVIOR AND FEELINGS OF PATIENTS WITH SYSTEMIC LUPUS ERYTHEMATOSUS DURING LOCKDOWN MEASURES FOR THE COVID-19 OUTBREAK}

G. De Marchi ${ }^{1}$, D. Azzolina ${ }^{2}$, E. Maresio ${ }^{3}$, D. Colatutto ${ }^{4}$, M. Binutti ${ }^{4}$, M. Monte ${ }^{5}$, S. Gallipoli ${ }^{6}$, F. Zobec ${ }^{6}$, A. Palese ${ }^{7}$, M. Silano ${ }^{8}$, S. De Vita ${ }^{4}$, D. Gregori ${ }^{9}$, L. Quartuccio ${ }^{4} .{ }^{1}$ ASUFC, Rheumatology Clinic, Udine, Italy; ${ }^{2}$ University of Piemonte Orientale, Statistics, Turin, Italy; ${ }^{3}$ ProChild Onlus, Psychology, Trieste, Italy; ${ }^{4}$ University of Udine, Rheumatology Clinic, Udine, Italy; ${ }^{5}$ Associazione Malati Reumatici - Friuli Venezia Giulia, Section - Udine, Udine, Italy; ${ }^{6}$ Zeta
Research srl, Clinical Project Management \& Quality Assurance, Trieste, Italy; ${ }^{7}$ University of Udine, School of Nursing, Department of Medicine, Udine, Italy; ${ }^{8}$ Istituto Superiore di Sanità, Unit of Human Nutrition and Health, Department of Food Safety Nutrition and Veterinary Public Health, Rome, Italy; ${ }^{9}$ University of Padova, Unit of Biostatistics, Epidemiology and Public Health, Department of Cardiology, Thoracic and Vascular Sciences, Padova, Italy

Background: COVID-19 is a systemic viral disease currently spreading as a pandemic. A more severe course and prognosis of COVID-19 in systemic lupus erythematosus (SLE) and vasculitis has been reported (1).

Several papers have focused on the concerns, healthcare-related behaviors and psychological impact of COVID-19 pandemic among patients with rheumatic diseases, and specifically on SLE patients, showing a trend towards remarkable psychological distress (2-4). To date, no investigation on the psychological effects of quarantine strategy on SLE patients has been carried out.

Objectives: To investigate the psychological impact of the lockdown measures adopted in Italy to contrasting the COVID-19 outbreak, on patients with SLE as compared to the general population.

Methods: Patients affected by SLE were given an online questionnaire focused on psychological impact and self-perception during the lockdown measures contrasting the COVID-19 outbreak. The survey was focused on COVID-19 concerns, emotional impact, self-perception and changes in daily living activities and relationships. Results were compared with those of PRESTO (imPact of quaRantine mEasures againST cOvid19) project, an Italian survey, which used the same questionnaire, directed to the general population, with or without chronic diseases. A propensity matching procedure has been applied to LEPRE (Lupus Erythematosus PRESTO project) cases and the PRESTO responders with a ratio of 2 versus 1 . Results: 64 patients and 1114 unselected people completed the survey. After the matching procedure, patients were compared to 128 matched adults. Missing data were below $6 \%$. The median age among patients was 43 years (I-III interquartile range $35-54.5$ ), $88 \%$ were female and $100 \%$ Caucasian. The SLE subjects live mainly in detached houses (38/64 vs $348 / 1114, p<0.0001)$, having access to a private garden $(52 / 64$ vs $625 / 1112, p<0.0001)$ and also owning a pet $(43 / 64$ vs $508 / 1114, p<0.001$ ), in comparison with the PRESTO sample living mainly in flats. The psychological impact measured by IES-R, GHQ.12, and CEDS scores were not statistically different between patients and the general population, such as globally COVID-19 concerns and feelings. However, patients perceived more difficulty to find some free time and enjoy it ( $13 / 60$ vs $48 / 121, p=0.01$ ) and to be able to solve own problems ( $47 / 61$ vs $71 / 120, p=0.02)$. On the contrary, patients felt more able to cope with the problem and less sad or depressed in comparison with the PRESTO group (17/61 vs $13 / 120, p=0.003)$. Moreover, patients missed playing sports/exercise less than general population ( $12 / 63$ vs $46 / 128, p=0.02$ ), while they felt more the distance from family and relatives ( $45 / 63$ vs $42 / 86, p<0.0001$ ).

Conclusion: the COVID-19 pandemic didn't unveil a greater psychological fragility of people living with SLE than the others. By contrast, a coping strategy, including the role of the family and the lifestyle, contributes to resilience of SLE in difficult scenarios such as those presented by the pandemic.

REFERENCES:

[1] Gianfrancesco M, et al. Ann Rheum Dis 2020;79:859-866.

[2] George M, et al. J Rheumatol 2020 Nov 15; doi: 10.3899/jrheum.201017.

[3] Rathi M, et al. Lupus 2020 Oct 6:961203320962855. doi 10.1177/0961203320962855

[4] Koppert TY, et al. Rheumatology 2020;keaa842. doi: 10.1093/rheumatology/ keaa842.

Acknowledgements: We thank the PRESTO Investigators dr Corrado Lanera and dr Giulia Lorenzoni (Biostatistics, Epidemiology and Public Health, Department of Cardiology, Thoracic and Vascular Sciences, University of Padova, Italy) for their contribution.

Disclosure of Interests: None declared

DOI: 10.1136/annrheumdis-2021-eular. 1590

\section{POS1193}

CLINICAL FEATURES AND THE COURSE OF COVID19 IN PATIENTS WITH FAMILIAL MEDITERRANEAN FEVER

A. Avanoglu Guler ${ }^{1}$, T. Yüce İnel ${ }^{2}$, H. Karadeniz ${ }^{1}$, R. Bilici Salman ${ }^{1}$, H. Satış ${ }^{1}$, H. Küçük' ${ }^{1}$ M. A. Ozturk' ${ }^{1}$ B. Goker ${ }^{1}$, S. Haznedaroglu' ${ }^{1}$ İ. Sari' ${ }^{2}$, T. Kasifoglu' ${ }^{3}$, A. Tufan'. ' Gazi University Faculty of Medicine Hospital, Rheumatology Department, Ankara, Turkey; ${ }^{2}$ Dokuz Eylul University Faculty of Medicine Hospital, Rheumatology Department, Izmir, Turkey: ${ }^{3}$ Eskisehir Osmangazi University Faculty of Medicine Hospital, Rheumatology Department, Eskisehir, Turkey

Background: The novel coronavirus 2019 (COVID-19) has a wide range of clinical presentation from asymptomatic or mild viral infection to severe life-threating complications, including acute respiratory distress syndrome (ARDS), which develop as a result of immune system dysregulation, exaggerated immune response, and cytokine release syndrome [1]. Familial Mediterranean Fever (FMF) is a hereditary autoinflammatory disorder characterized by dysfunction 
of the innate immune system and excessive production of proinflammatory cytokines, including interleukin (IL)-1 $\beta$, IL-6, interferon-gamma, and tumor necrosis factor-alpha, all of which have increased in severe cases in COVID-19 [1-3]. Objectives: The aim of this study is to report clinical characteristics and outcome of FMF patients with COVID-19.

Methods: This study included 48 consecutive FMF patients who were diagnosed COVID-19 by SARS-CoV-2 nucleic acid RT-PCR in nasopharyngeal swab or sputum, or symptoms and computed tomography findings suggestive for COVID-19. Data on demographic and clinical characteristics of FMF disease, clinical course and outcome of COVID-19 were evaluated.

Results: The median age of patients was 35.5 (29-43.5) years, ranging from 18 to 87 years. The median disease duration of FMF was $10(6-16)$ years. The most common presenting symptoms and signs of patients during attacks were peritonitis $(85.5 \%)$, fever $(81.3 \%)$, and pleuritis $(48 \%)$. Twelve $(25 \%)$ patients had amyloidosis. Comorbidities were present in half of patients with ankylosing spondylitis $(21 \%)$ and hypertension (17\%) being the most frequently seen. Two-third of patients $(66 \%)$ were in remission for FMF. The median dosage of colchicine treatment for FMF was 1.5 (IQR 1) mg/day. $90 \%$ of patients continued colchicine treatment for FMF during the COVID-19 course. The baseline characteristics and treatment modalities of patients were demonstrated in Table 1. Forty-six patients presented with at least one COVID-19 symptoms. Fever (73\%), myalgia/arthralgia $(69 \%)$, and cough $(60 \%)$ were the most common symptoms. 16 patients were admitted at hospital, 5 of them required oxygen therapy and 2 patients with amyloidosis developed ARDS and went to intensive care unit (ICU) for invasive mechanical ventilation (IMV). One patient who had been followed-up at ICU died. Conclusion: Our FMF patients with COVID-19 have similar clinical features and outcomes as general population. Dysregulation of innate immune system in FMF might not be risk factors for COVID-19. Besides, colchicine and IL-1 inhibitors intake might have protective and preventive effects on COVID-19 progression.

Table 1. Baseline characteristics, treatment modalities and outcome of patients

\begin{tabular}{|c|c|}
\hline Age years, median (IQR) & $39.4(15)$ \\
\hline Gender (F/M) & $25 / 23$ \\
\hline FMF Disease duration, years, median (IQR) & $10(10)$ \\
\hline \multicolumn{2}{|l|}{ Mutations ${ }^{*}$} \\
\hline Monoallelic mutation, $\mathrm{n}(\%)$ & $6(14)$ \\
\hline Biallelic mutation, $\mathrm{n}(\%)$ & $34(81)$ \\
\hline Homozygous M694V mutation & $18(43)$ \\
\hline Heterozygous M694V mutation & $15(36)$ \\
\hline Comorbidities, n (\%) & $24(50)$ \\
\hline \multicolumn{2}{|l|}{ FMF treatment } \\
\hline Colchicine $1 \mathrm{mg} /$ day, $\mathrm{n}(\%)$ & $17(35.5)$ \\
\hline Colchicine $1.5 \mathrm{mg} /$ day, $\mathrm{n}(\%)$ & $18(37.5)$ \\
\hline Colchicine $2 \mathrm{mg} /$ day, $\mathrm{n}(\%)$ & $13(27)$ \\
\hline Anakinra, $\mathrm{n}(\%)$ & $12(25)$ \\
\hline Canakinumab, n (\%) & $3(6.3)$ \\
\hline Positive SARS-Cov-2 RT-PCR, n (\%) & $42(87.5)$ \\
\hline Interstitial pneumonia in CT scan, $\mathrm{n}(\%)$ & $15(31.3)$ \\
\hline COVID-19 treatment, $\mathrm{n}(\%)$ & $46(95.8)$ \\
\hline Outpatient treatment, $\mathrm{n}(\%)$ & $32(66.7)$ \\
\hline Hospitalized, not required supplemental oxygen, n (\%) & $9(18.8)$ \\
\hline Hospitalized, required supplemental oxygen, $n$ (\%) & $5(10.4)$ \\
\hline ICU, required IMV, n (\%) & $2(4.2)$ \\
\hline \multicolumn{2}{|l|}{ Outcome } \\
\hline Recovered, n (\%) & $47(98)$ \\
\hline Deceased, $\mathbf{n}(\%)$ & $1(2)$ \\
\hline Complications, $\mathbf{n}(\%)$ & $3(6.3)$ \\
\hline
\end{tabular}

*42 patients were included in the analysis

\section{REFERENCES:}

[1] Tufan A, Avanoğlu Güler A, Matucci-Cerinic M. COVID-19, immune system response, hyperinflammation and repurposing antirheumatic drugs. Turkish journal of medical sciences. 2020;50:620-632.

[2] Hausmann JS. Targeting cytokines to treat autoinflammatory diseases. Clinical immunology (Orlando, Fla.). 2019;206:23-32.

[3] Tufan A, Lachmann HJ. Familial Mediterranean fever, from pathogenesis to treatment: a contemporary review. Turkish journal of medical sciences. 2020;50:1591-1610.

Disclosure of Interests: None declared.

DOI: 10.1136/annrheumdis-2021-eular.1649

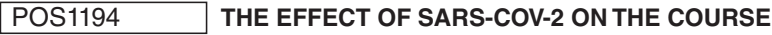 AND THE TREATMENT OF RHEUMATIC INFLAMMATORY DISEASES. EXPERIENCE FORM THE NORTHWESTERN GREECE}

E. Pelechas ${ }^{1}$, E. Kaltsonoudis ${ }^{1}$, M. Migos ${ }^{1}$, P. Karagianni ${ }^{2}$, A. Kavvadias ${ }^{3}$, P. Voulgari ${ }^{1}$, A. Drosos ${ }^{1} .{ }^{1}$ University of loannina, School of Medicine, Greece, Internal Medicine - Division of Rheumatology, loannina, Greece; ${ }^{2}$ University of loannina, School of Medicine, Greece, Laboratory Medicine, Ioannina,
Greece; ${ }^{3}$ University of loannina, School of Medicine, Greece, Gastroenterology, loannina, Greece

Background: COVID-19 has been shown to significantly affect the vulnerable population [1,2]. Among them, patients suffering from inflammatory rheumatic diseases, and especially the immunosuppressed [3].

Objectives: to assess the effect of SARS-CoV-2 on the course and the treatment of rheumatic inflammatory diseases.

Methods: from February to December 2020, 46 patients with inflammatory rheumatic diseases were included (32 female) that got infected with the SARS-CoV-2 Mean age was 65 years old, 17 were smokers, 12 had arterial hypertension, 8 diabetes mellitus, and 3 hypothyroidism. Most of them had their comorbidities well-controlled and their rheumatic disease was in remission. More specifically, 24 patients had rheumatoid arthritis, 13 psoriatic arthritis, and 9 ankylosing spondylitis. All patients were under treatment with conventional synthetic (cs) and/or biological (b) disease-modifying anti-rheumatic drugs (DMARDs), while 7 of them were also on treatment with glucocorticoids (GC) $(<5 \mathrm{mg} /$ day). Twenty-eight patients were on tumor necrosis alpha (TNF- $\alpha$ ) inhibitors (19 as monotherapy), 4 on anti- interleukin (IL)- 6 monotherapy, 3 on Janus Kinase (JAK) inhibitors plus on low dose methotrexate (MTX), and the rest (11 patients) were on a cSDMARD with or without GCs.

Results: positive patients with the SARS-CoV-2, instructed to discontinue their immunosuppressive treatment, except GCs that were adjusted for their disease. Most patients (37 out of 46 ) had a mild disease course and their symptomatology was nothing more than a simple flu-like syndrome. Furthermore, on 9 of them olfactory dysfunction and gastrointestinal manifestations as well as low grade fever were noted but without the need of a hospital admission. On the other hand only 5 patients needed hospitalization ( 2 on MTX monotherapy and 3 on combination therapy) due to dyspnea with low oxygen saturation (hypoxemia) and high fever. From those 5, 3 had a short in-hospital stay, while 2 developed pneumonia and a longer in-hospital stay was required in order to get the appropriate treatment. None of the patients did not require an intensive care unit admission. Finally, in 14 patients that got infected from February to May 2020, viral antibodies had been measured. All patients had high titres of IgG antibodies in their serum for as long as six months after their infection. Of note, none of the infected patients were smokers.

Conclusion: patients with rheumatic diseases that are in remission using low doses of GCs and DMARDs, have almost the same chances with the genera population to have a serious course of their infection with the SARS-Cov-2. In addition, in these patients, the immune response appears to be adequate, both in the production and maintenance of antibodies, which appear to be maintained for at least 6 months after infection.

\section{REFERENCES:}

[1] Patel JA, Nielsen FBH, Badiani AA, Assi S, Unadkat VA, Patel B, et al Poverty, inequality and COVID-19: the forgotten vulnerable. Public Health. 2020;183:110-111. Doi: 10.1016/j.puhe.2020.05.006

[2] Poteat T, Millet GA, Nelson LE, Beyrer C. Understanding COVID-19 risks and vulnerabilities among black communities in America: the lethal force of syndemics. Ann Epidemiol. 2020;47:1-3. Doi: 10.1016/j.annepidem.2020.05.004

[3] Gianfrancesco MA, Hyrich KL, Gossec L, Strangfeld A, Carmona L, Mateus $E F$, et al. Rheumatic disease and COVID-19: initial data from the COVID19 Global Rheumatology Alliance provider registries. Lancet Rheumatol. 2020;2(5):e250-e253. Doi: 10.1016/S2665-9913(20)30095-3.

Disclosure of Interests: None declared.

DOI: 10.1136/annrheumdis-2021-eular.1691

\section{POS1195 ASSESSING ANTIBODY STATUS FOR SARS-CoV-2 IN PEOPLE WITH CORONAVIRUS INFECTION: A TIME COURSE STUDY IN PEOPLE WITH AUTOIMMUNE CONDITIONS}

K. Biddle ${ }^{1}$, S. Koushesh ${ }^{1}$, A. Blundell ${ }^{1}$, D. Clark ${ }^{1}$, S. Krishna ${ }^{1}$, N. Sofat ${ }^{1} .{ }^{1}$ St George's University of London, Institute for Infection and Immunity, London, United Kingdom

Background: With the emergence of the global coronavirus pandemic, increas ing concerns have been raised about the course of SARS-CoV-2 infection in people with immune-mediated disorders.

Objectives: In this study we aimed to assess the time course of proven SARSCoV-2 infection, development of humoral immunity with detectable antibodies to the virus and evaluate any changes in antibody titres over time.

Methods: We recruited 114 participants in total who had potential symptoms of Covid-19 infection. Participants were recruited from rheumatology or inflammatory bowel disease (IBD) clinics from their records who attended a London Teaching hospital for care. Ethical Approval was in place for the study. Age- and gender- matched control participants without any underlying rheumatological condition/IBD were recruited as a comparator group. Clinical symptoms fo Covid-19 infection were assessed using the Covid-19 Rheumatology Global 